\title{
Appendix to Plane Sampling for Light Paths from the Environment Map
}

\author{
Holger Dammertz and Johannes Hanika \\ \{holger.dammertz,johannes.hanika\}@uni-ulm.de \\ Ulm University
}

\begin{abstract}
After publication of our corresponding article [DH09] in the journal of grahics, games, and gpu tools, we received questions about the precise mathematical formulation, especially the geometric terms. We hope to clarify this by giving an appendix which contains an additional mathematical explanation.
\end{abstract}

\section{A. Mathematical Formulation}

Monte Carlo methods for light transport estimate integrals using the expected value of some constructed random variable, e.g. to evaluate how much light emitted from a surface patch $A_{y}$ is received by a surface patch $A_{x}$ :

$$
\begin{aligned}
F & =\int_{A_{x}} \int_{A_{y}} L(y \leftrightarrow x) \underbrace{\frac{\cos _{x} \cos _{y}}{\|x-y\|^{2}}}_{=: G} V(x \leftrightarrow y) d y d x \\
& =\int_{A_{x}} \int_{S_{-}^{2}} L(x \leftarrow \omega) \cos _{x} d \omega d x \\
& =\int_{A_{y}} \int_{S^{2}} L(y \rightarrow \omega) V(x \leftrightarrow y) \cos _{y} d \omega d y,
\end{aligned}
$$

which can be evaluated by corresponding Monte Carlo estimators, each sampling a combination of $x, y$ and $\omega$ for the respective integrals. The first line would sample two points and directly evaluate the geometric term $G$. Equation 1 describes a backward path tracer, sampling an outgoing direction from $x$ (see Figure 1). Equation 2 describes a light tracer, starting with a point $y$ on the light source. This last variant is especially useful to render caustics and the starting point for many algorithms (e.g. photon mapping). 

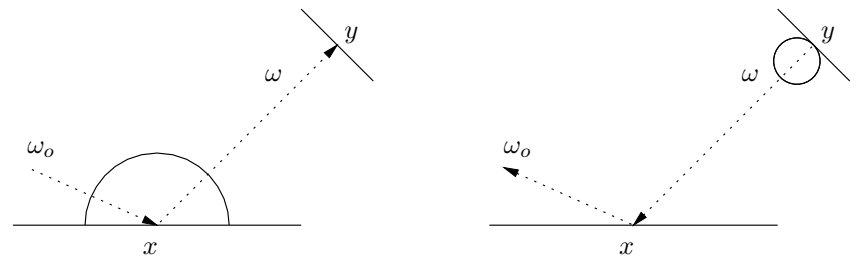

Figure 1: Different densities for light source sampling: sampling the hemisphere $p\left(\omega_{i}\right)=\frac{1}{2 \pi}$ (left), sampling the area of the light source $p(y)=\frac{1}{A_{l}}$ (middle), sampling a starting point and direction of a light ray on the environment map $p\left(\omega_{i}\right)=\frac{1}{4 \pi}, p(y)=\frac{1}{A}$ (right).

The corresponding estimator using Equation 2 is

$$
F=E\left(\frac{1}{N} \sum_{k=1}^{N} \frac{L\left(y_{k} \rightarrow \omega_{k}\right) V \cos _{y_{k}}}{p\left(y_{k}\right) p\left(\omega_{k}\right)}\right)=E\left(\frac{1}{N} \sum_{k=1}^{N} \pi\left|A_{y}\right| L\left(y_{k} \rightarrow \omega_{k}\right) V\right)
$$

with $p\left(y_{k}\right)=1 /\left|A_{y}\right|$ a uniform distribution on the surface of the light source and $p\left(\omega_{k}\right)=\cos _{y_{k}} / \pi$ following a cosine distribution. For image-based lighting, it is not as straightforward to find these probability densities. While $p(\omega)$ can easily be modelled (e.g. by using $p(\omega)=1 /(4 \pi)$ uniformly on the sphere), the question remains where to put $y$.

To allow for light tracing from an environment map, we start by reformulating a similar expression to evaluate how much light emitted from an environment map is received by a surface patch $A_{x}$ :

$$
\begin{aligned}
F_{\text {env }} & =\int_{A_{x}} \int_{\Omega} L(x \leftarrow \omega) \cos _{x} d \omega d x \\
& =\int_{\Omega} \int_{A_{x}} L(x \leftarrow \omega) \underbrace{\cos _{x} d x}_{=: d y} d \omega \\
& =\int_{\Omega} \int_{A_{y}} L(y \rightarrow \omega) V(x \leftrightarrow y) d y d \omega \\
& =E\left(\frac{1}{N} \sum_{k=1}^{N} \frac{L\left(y_{k} \rightarrow \omega_{k}\right) V}{p\left(y_{k}\right) p\left(\omega_{k}\right)}\right) .
\end{aligned}
$$

Equation 4 in fact describes a light tracing algorithm. The newly introduced variable $y=f(x)+C$ with $\left|J_{f}\right|=\cos _{x}$ can be found geometrically on a plane perpendicular to $\omega$ (see Figure 2). The vector $C$ is used to shift the 


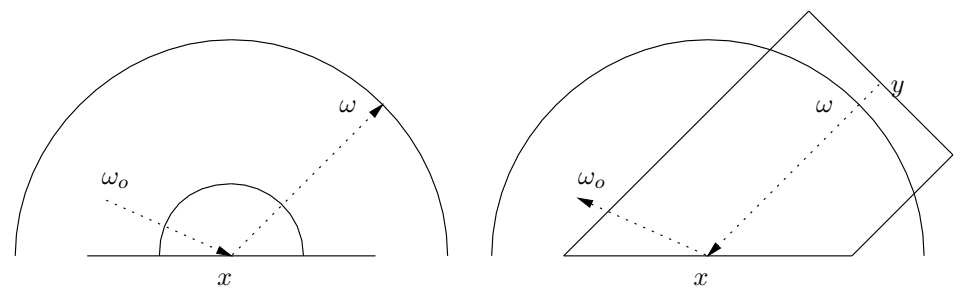

Figure 2: Sampling densities for image-based lighting: path tracing, as in Equation 3 (left) and light tracing, as in Equation 4 (right).

plane out of the bounding box, so the visibility term $V$ will be equivalent to the one evaluated for the environment map.

This principle is easily extended from one surface patch $A_{x}$ to the whole scene. The random variable $y$ remains the same, even for different surface normals resulting in different $\cos _{x}$, as the substitution explicitly removes this term. The visibility term $V(x \leftrightarrow y)$ takes care of occlusion and even allows for a too large integration domain $A_{y}$, i.e. which covers more than just $A_{x}$ after the transformation.

To start paths on the environment map, the densities for $y$ and $\omega$ are separated, as for geometry light source sampling. Let's assume for the moment that $p(\omega)=\frac{1}{4 \pi}$ uniformly on the sphere will be sufficient. So at this stage we have a directional light source with direction $\omega$ and need to pick a starting point $y$. As such a light source has infinite extent or is infinitely far away, the first idea is to sample the end point $x$ for the light ray. It is very hard for complex scenes to find the correct density for such an end point. We thus use virtual planes of finite extent just outside the bounding box of the scene to represent all of the directional light which is of interest for the scene (see Figure 2, right).

Acknowledgments. The authors would like to thank the reviewers for their helpful suggestions. Additionally they want to thank mental images $\mathrm{GmbH}$ for funding parts of this research, and Matthias Raab, Leonhard Grünschloß and Daniel Seibert for many discussions.

\section{References}

[DH09] Holger Dammertz and Johannes Hanika. Plane sampling for light paths from the environment map. Journal of graphics, gpu and game tools, 14(2):25-31, 2009. 